\title{
Endothelial monocyte activating polypeptide II interferes with VEGF-induced proangiogenic signaling
}

\author{
Niranjan Awasthi ${ }^{1}$, Margaret A Schwarz ${ }^{1}$, Varun Verma², Clint Cappiello ${ }^{2}$ and Roderich E Schwarz ${ }^{1}$
}

Endothelial monocyte activating polypeptide II (EMAP II) is a proinflammatory cytokine with antiangiogenic properties. EMAP II functions as a potent inhibitor of primary and metastatic tumor growth, has strong inhibitory effects on endothelial cells (ECs), and can reduce intratumoral expression of the angiogenesis inducer vascular endothelial growth factor (VEGF). VEGF influences EC functions such as proliferation, migration, survival and tube formation. Therapeutic strategies that target VEGF have been demonstrated to reduce the tumor growth. We investigated the effects of EMAP II on VEGF-induced angiogenesis signaling. Primary human fetal lung ECs (HFLECs) and human umbilical vein ECs (HUVECs) were grown in E-Stim medium. Protein binding was analyzed using enzyme-linked immunosorbent assay (ELISA). Protein expression was determined by western blot analysis. EC proliferation and migration was determined using WST-1 reagent and transwell membrane, respectively. EMAP II efficiently and dose dependently binds to VEGF receptor 1 (VEGFR1) and VEGF receptor 2 (VEGFR2) as observed by ELISA. $B_{\max }$ values for VEGFR1 and VEGFR2 were 0.45 and 0.17 , respectively. In addition, EMAP II inhibited binding of VEGF to VEGFR1 and VEGFR2. EMAP II significantly reduced VEGF-induced expression of phosphorylated VEGFR1 (in HFLEC and HUVEC) by $>50 \%$, and of phosphorylated VEGFR2 (in HUVEC) by $66 \%$. EMAP II also inhibited downstream VEGF signaling. Although VEGF-induced phosphorylation of Akt, Erk1/2, p38 and Raf 2.8-, 1.5-, 2.2- and 3.6-fold, respectively, EMAP II preincubation blocked this induction in phosphorylation to control levels. VEGF-induced EC proliferation 2.5-fold, and EMAP II pretreatment abrogated this effect. Similarly, VEGF-induced EC migration (2.5-fold) was significantly inhibited by EMAP II. These finding suggest that inhibition of VEGF signaling is one possible antiangiogenic mechanism of EMAP II, which may explain its in vivo antitumor activity and delineate therapeutic strategies to enhance anti-VEGF therapy to inhibit tumor growth.

Laboratory Investigation (2009) 89, 38-46; doi:10.1038/labinvest.2008.106; published online 10 November 2008

KEYWORDS: angiogenesis; EMAP II; endothelial cells; migration; proliferation; VEGF inhibition

Endothelial monocyte activating polypeptide II (EMAP II) was first isolated from supernatants of cultured murine methylcholanthrene A-induced fibrosarcoma cells based on its capacity to induce tissue factor expression in human umbilical vein endothelial cells (HUVECs). ${ }^{1}$ EMAP II is a proinflammatory cytokine with antiangiogenic properties, which exerts pleiotropic effects on endothelial cells (ECs), monocytes and neutrophils. ${ }^{1}$ EMAP II is synthesized as a $34 \mathrm{kDa}$ pro-EMAP II, which is proteolytically cleaved to the $22 \mathrm{kDa}$ mature EMAP II (EMAP II), which has most of the described biological activities. ${ }^{2,3}$ Expression of pro-EMAP II and release of EMAP II has been shown to be increased in response to stress, such as hypoxia, treatment with chemotherapeutic drugs and induction of apoptosis. ${ }^{4,5}$ EMAP II has potent effects on ECs including release of von Willebrand factor; manipulation of coagulation properties; induced expression of tissue factor, E-selectin, P-selectin, DOC; $;^{1,2,6}$ and blocking adhesion of ECs to fibronectin as well as matrix assembly by binding to $\alpha 5 \beta 1$ integrin. ${ }^{7}$ Although EMAP II sensitizes tumors to the antitumor effects of tumor necrosis factor- $\alpha^{8,9}$ by inducing the expression of tumor necrosis factor- $\alpha$ receptor- $1,{ }^{10}$ it has in its own right been shown to induce apoptosis of ECs and inhibits proliferation, vascularization and neovessel formation. ${ }^{11,12}$ In addition, EMAP II has been shown to suppress primary and metastatic tumor growth by antiangiogenic properties ${ }^{11}$ and to reduce vascular endothelial growth factor (VEGF) expression, ${ }^{13}$ which itself facilitates tumor growth through induction of angiogenesis.

\footnotetext{
'Department of Surgery, University of Texas Southwestern Medical Center, UT Southwestern School of Medicine, Dallas, TX, USA and ${ }^{2}$ Department of Surgery, UMDNJ_Robert Wood Johnson Medical School, New Brunswick, NJ, USA

Correspondence: Dr RE Schwarz, MD, PhD, Department of Surgery, University of Texas Southwestern Medical Center, UT Southwestern School of Medicine, 5323 Harry Hines Boulevard, Dallas, TX 75390-8548, USA.

E-mail: roderich.schwarz@utsouthwestern.edu

Received 17 May 2008; revised 22 August 2008; accepted 27 September 2008
} 
Angiogenesis, the formation of new blood vessels, be involved in tumor growth and metastasis. ${ }^{14,15}$ Angiogenesis is a complex process and involves the survival, activation, proliferation, differentiation, migration and reorganization of ECs. ${ }^{15,16}$

VEGF is an EC-specific mitogen, and its high affinity receptor-binding sites are located on ECs. ${ }^{17}$ VEGF has shown to be expressed in a wide variety of tumors. VEGF is the most important mediator of angiogenesis associated tumor development as it regulates survival, proliferation, differentiation, migration, permeability and tube formation of ECs. ${ }^{18-21}$ VEGF also functions as an antiapoptotic factor for ECs in newly formed vessels. ${ }^{22}$ Several reports in the literature have shown that therapeutic strategies involving VEGF inhibition, by either targeting VEGF or its receptors, decrease vessel density and reduce tumor growth. ${ }^{23-25}$ VEGF activates its intracellular signaling pathway by binding with high affinity to two tyrosine kinase receptors, VEGF receptor 1 (VEGFR1/Flt-1) and VEGF receptor 2 (VEGFR2/Flk-1), ${ }^{26}$ that induce receptor dimerization and subsequent induction of kinase activity and transphosphorylation of several cytoplasmic signaling proteins including mitogen-activated protein kinase (MAPK), extracellular signal-regulated protein kinase (ERK), stress-activated protein kinase (p38 MAP kinase) and phosphatidylinositol 3-kinase. ${ }^{27}$

In this study, we observed that EMAP II interferes with the VEGF-induced angiogenic signaling pathway, suggesting that this may be one of the antiangiogenic mechanisms of EMAP II towards preventing tumor growth and metastasis.

\section{MATERIALS AND METHODS}

\section{Cell Culture and Treatments}

Primary human fetal lung ECs (HFLECs) were a generous gift from Dr MJ Acarregui (University of Iowa, Iowa City, IA, USA) and were grown on type I collagen (BD Biosciences, Bedford, MA, USA) coated plates. HUVECs were grown on $1 \%$ gelatin (Sigma-Aldrich, St Louis, MO, USA) coated plates. Both of these cell lines were grown in E-Stim medium supplemented with epidermal growth factor and $\mathrm{EC}$ growth supplement (BD Biosciences) at $37^{\circ} \mathrm{C}$ in a humidified $5 \%$ $\mathrm{CO}_{2}$ atmosphere. All the cells were used within passage three to seven where they showed specific characteristics of ECs.

Monolayers of cells at $75-80 \%$ confluence were serum starved for at least $5 \mathrm{~h}$ before treatment with recombinant human VEGF 165 (Sigma) and EMAP II. For western blot analysis, cells were treated with $20 \mathrm{ng} / \mathrm{ml}$ VEGF, $20 \mu \mathrm{M}$ EMAP II or preincubated with EMAP II for $30 \mathrm{~min}$ and then treated with VEGF. In proliferation assays, cells were treated with EMAP II $(10$ and $20 \mu \mathrm{M})$ and VEGF $(1,10$ or $20 \mathrm{ng} / \mathrm{ml})$ over $72 \mathrm{~h}$. In cotreated samples, cells were preincubated with EMAP II for $30 \mathrm{~min}$ and then VEGF was added. For migration assay, $300 \mu \mathrm{l}$ cell suspension (from $0.3 \times 10^{6}$ cells per $\mathrm{ml}$ ) was added into the upper filter chamber of dual chamber wells with or without preincubation with EMAP II, whereas
$20 \mathrm{ng} / \mathrm{ml}$ VEGF or $20 \mu \mathrm{M}$ EMAP II were added into the lower chamber.

\section{Production and Purification of Recombinant EMAP II}

Recombinant EMAP II was prepared from E. coli transformed with a plasmid containing a $6 \times$ his-tag and the coding sequence for EMAP II, as previously described. ${ }^{28}$ Recombinant EMAP II protein was purified using Ni-NTA columns under native conditions as per manufacturer's protocol with all procedures performed at $4^{\circ} \mathrm{C}$ (Qiagen, Valencia, CA, USA). Briefly, frozen $E$. coli cell pellets were lysed in buffer containing $50 \mathrm{mM} \mathrm{NaH} \mathrm{PO}_{4}, 300 \mathrm{mM} \mathrm{NaCl}, 10 \mathrm{mM}$ imidazole, protease inhibitor cocktail, $\beta$-mercaptoethanol and $1 \mathrm{mg} / \mathrm{ml}$ lysozyme. Cell lysate was then sonicated and centrifuged at $12000 \mathrm{rpm}$ for $20 \mathrm{~min}$, and supernatant was mixed gently with $50 \%$ Ni-NTA slurry. The lysate-Ni-NTA mixture was loaded into a column and washed with buffer containing $50 \mathrm{mM} \mathrm{NaH} \mathrm{PO}_{4}, 300 \mathrm{mM} \mathrm{NaCl}$ and $20 \mathrm{mM}$ imidazole. After washing, EMAP II protein was eluted with buffer containing $50 \mathrm{mM} \mathrm{NaH} \mathrm{PO}_{4}, 300 \mathrm{mM} \mathrm{NaCl}$ and $250 \mathrm{mM}$ imidazole. EMAP II protein was then dialyzed against PBS, aliquoted and stored at $-80^{\circ} \mathrm{C}$.

\section{Enzyme-Linked Immunosorbent Assay}

Ninety six-well plates were coated with $800 \mathrm{nM}$ of EMAP II in $50 \mu \mathrm{l}$ carbonate-bicarbonate buffer ( $\mathrm{pH} 9.6)$ per well at $4^{\circ} \mathrm{C}$ overnight. Wells were then washed with PBS containing $0.02 \%$ Tween 20 and blocked with $1 \%$ BSA in PBS for $30 \mathrm{~min}$ at $37^{\circ} \mathrm{C}$. Increasing concentrations $(1,3,10,30$ and $100 \mathrm{ng} / \mathrm{ml})$ of VEGFR1 or VEGFR2 were added to EMAP II coated wells and plates were incubated overnight at $4{ }^{\circ} \mathrm{C}$. To investigate the effect of EMAP II on binding of VEGF to its receptors VEGFR1 and VEGFR2, wells were coated with $250 \mathrm{ng} / \mathrm{ml}$ of VEGF in $50 \mu \mathrm{l}$ coating buffer and incubated overnight at $4^{\circ} \mathrm{C}$. After washing, wells were blocked with $1 \%$ BSA for $30 \mathrm{~min}$ at $37^{\circ} \mathrm{C}$. VEGFR1 or VEGFR2 $(50 \mathrm{ng} / \mathrm{ml})$ was preincubated with EMAP II $(4,40,120$ and $400 \mathrm{nM})$ for $30 \mathrm{~min}$ at $4^{\circ} \mathrm{C}$ and then added to VEGF coated wells and incubated overnight at $4^{\circ} \mathrm{C}$. After this step, for both of the above experiments, wells were washed and incubated for $1 \mathrm{~h}$ with anti-VEGFR1 antibody (Chemicon, Temecula, CA, USA) or anti-VEGFR2 antibody (R\&D Systems) at $37^{\circ} \mathrm{C}$ with shaking. After washing, an HRP-conjugated secondary antibody was added and incubated for $1 \mathrm{~h}$ at $37^{\circ} \mathrm{C}$ with shaking. After washing, $100 \mu \mathrm{l}$ of $o$-phenylenediamine chromogenic substrate (Sigma) was added to each well and the plate was incubated for $15 \mathrm{~min}$ in the dark. The reaction was stopped by the addition of $100 \mu \mathrm{l}$ $2 \mathrm{M} \mathrm{H}_{2} \mathrm{SO}_{4}$ and absorbance was read at $490 \mathrm{~nm}$.

\section{Western Blot Analysis}

Cells were lysed in buffer containing $20 \mathrm{mM}$ Tris-HCl, $150 \mathrm{mM} \mathrm{NaCl}, 1 \%$ Triton X-100, 0.05\% NP-40, $50 \mathrm{mM} \mathrm{NaF}$, $1 \mathrm{mM}$ sodium orthovanadate, $5 \mathrm{mM}$ sodium pyrophosphate, $1 \mathrm{mM}$ PMSF, $10 \mu \mathrm{l}$ each of protease and phosphatase inhibitor per $\mathrm{ml}$ of lysis buffer; and incubated for $20 \mathrm{~min}$ at $4^{\circ} \mathrm{C}$ 
with gentle shaking. The lysate was centrifuged for $15 \mathrm{~min}$ at $13000 \mathrm{rpm}$, supernatant protein concentrations were measured and equal amount of total protein were separated by SDS-PAGE and transferred to PVDF membranes (BioRad, Hercules, CA, USA). The membranes were blocked for $1 \mathrm{~h}$ at room temperature with gentle shaking in TBS-T (10 mM Tris- $\mathrm{HCl}$ (pH 7.6), $150 \mathrm{mM} \mathrm{NaCl}, 0.05 \%$ Tween 20) containing 5\% nonfat milk. The membranes were then incubated overnight at $4^{\circ} \mathrm{C}$ with the corresponding antibodies: anti-Akt and antiphospho-Akt, anti-Erk (all from Cell Signaling Technology, Danvers, MA, USA) and antiphosphoErk, anti-p38 and antiphospho-p38, anti-Raf- 1 and antiphospho-Raf-1 (all from Santa Cruz Biotechnologies, Santa Cruz, CA, USA), anti-VEGFR1 (Chemicon) and antiphospho-VEGFR1 (Upstate Biotechnology, Lake Placid, NY, USA), anti-VEGFR2 (Cell Signaling) and antiphosphoVEGFR2 (Upstate) or anti $\alpha$-tubulin (Sigma). These blots were then incubated with corresponding HRP-conjugated secondary antibodies (Pierce Biotechnology, Rockford, IL, USA) for $1 \mathrm{~h}$ at room temperature, and specific bands were detected using the enhanced chemiluminescence reagent (PerkinElmer Life Sciences, Boston, MA, USA) on autoradiographic film. Protein bands were quantitated by densitometry, and protein loading was normalized with $\alpha$-tubulin.

\section{Cell Proliferation Assay}

The proliferation of primary ECs (HFLEC and HUVEC) was evaluated by an assay employing the sulfonated tetrazolium salt WST-1 (4-[3-(4-iodophenyl)-2-(4-nitrophenyl)-2H-5tetrazolio]-1,3-benzene disulfonate). The measurement is based on the ability of viable cells to cleave tetrazolium salts by mitochondrial dehydrogenases. Briefly, 4000 cells per well were plated in a 96-well microplate in regular supplemented E-Stim EC growth medium and incubated at $37^{\circ} \mathrm{C}$ and $5 \%$ $\mathrm{CO}_{2}$. After a 16-h incubation, the medium was removed, cells were washed twice with PBS and Basal EC medium (EBM-2 from Cambrex BioScience, Wakersville, MD, USA) was added in each well. After $5 \mathrm{~h}$ incubation, cells were treated with EMAP II and VEGF, either alone or in combination, and incubated for $72 \mathrm{~h}$ at $37^{\circ} \mathrm{C}$ and $5 \% \mathrm{CO}_{2}$. To assay for proliferation, $10 \mu \mathrm{l}$ per well WST-1 reagent was added and after $2 \mathrm{~h}$ incubation, absorbance of the samples was measured at $450 \mathrm{~nm}$ using a microplate reader with a blank as the background control.

\section{Cell Migration Assay}

The cell migration assay was performed using a CytoSelect Cell Migration assay kit (Cell Biolabs Inc., San Diego, CA, USA). Briefly, HUVECs grown up to $75-80 \%$ confluence were serum starved for $5 \mathrm{~h}$, trypsinized and suspended in EBM-2 medium at a final concentration of $0.3 \times 10^{6}$ cells per $\mathrm{ml}$. In 24 -well plate, $300 \mu \mathrm{l}$ of this cell suspension was added to the upper filter chamber with polycarbonate membrane ( $8 \mu \mathrm{m}$ pore size) with or without preincubation with $20 \mu \mathrm{M}$ EMAP II for $30 \mathrm{~min}$. In lower chamber $500 \mu \mathrm{l}$ of serum-free medium was added containing 10\% FBS (positive control), $20 \mathrm{ng} / \mathrm{ml}$ VEGF or $20 \mu \mathrm{M}$ EMAP II. The plate was incubated at $37^{\circ} \mathrm{C}$ and $5 \% \mathrm{CO}_{2}$ for $24 \mathrm{~h}$. After incubation, nonmigratory cells on the upper surface of the filter were removed by wiping with a cotton swab, and migratory cells were stained, photographed under inverted light microscope, and relative migration was quantitated by measuring absorption at $560 \mathrm{~nm}$.

\section{Statistical Analysis}

Statistical significance was analyzed using GraphPad Prism 4 Software (GraphPad Software, San Diego, CA, USA). Results are expressed as mean \pm standard deviation (s.d.). Data were analyzed by the two-tailed Student's $t$-test and values of $P<0.05$ were accepted as reflecting statistically significant group differences.

\section{RESULTS \\ EMAP II Binds with VEGF Receptors}

In an enzyme-linked immunosorbent assay (ELISA) experiment, recombinant EMAP II was bound to the plate and was allowed to interact with increasing concentrations of recombinant VEGFR1 or VEGFR2. We observed a dose-dependent increase in the binding of EMAP II to both VEGFR1 and VEGFR2, which was saturable (Figure 1). Data were subjected to Scatchard analysis to determine maximum binding $\left(B_{\max }\right)$ and equilibrium constant $\left(K_{\mathrm{D}}\right)$. The $B_{\max }$ and $K_{\mathrm{D}}$ values for VEGFR1 were 0.45 and 4.43 , and for VEGFR2 were 0.17 and 9.63, respectively, indicating a higher affinity of EMAP II for VEGFR1 compared to VEGFR2. A similar binding pattern was observed when VEGFR1 or VEGFR2 were bound to the plate and were allowed to interact with increasing concentrations of EMAP II (data not shown). We also observed that EMAP II did not bind to VEGF protein (data not shown).

\section{EMAP II Inhibits Binding of VEGF to Its Receptors}

To investigate if EMAP II preincubated with VEGFR can interfere with the binding of VEGF to its receptors, we performed an ELISA to observe the effect of EMAP II on binding of VEGF to its receptors VEGFR1 and VEGFR2. In this experiment, EMAP II was preincubated with VEGFR1 or VEGFR2 at increasing concentrations, before allowing it to interact with VEGF bound to the plate. We observed that EMAP II preincubated with VEGFR at increasing doses could decrease the binding of VEGF with its receptors VEGFR1 and VEGFR2. At a $400 \mathrm{nM}$ concentration of EMAP II, the binding of VEGF with VEGFR1 and VEGFR2 was decreased by approximately 53 and $42 \%$, respectively (Figure 2).

\section{EMAP II Inhibits VEGF-Induced Phosphorylation of VEGFR1 and VEGFR2}

After observing the interaction of EMAP II with VEGFR1 and VEGFR2 by ELISA, we investigated the effect of EMAP II on VEGF-induced phosphorylation of VEGFR1 and VEGFR2. 

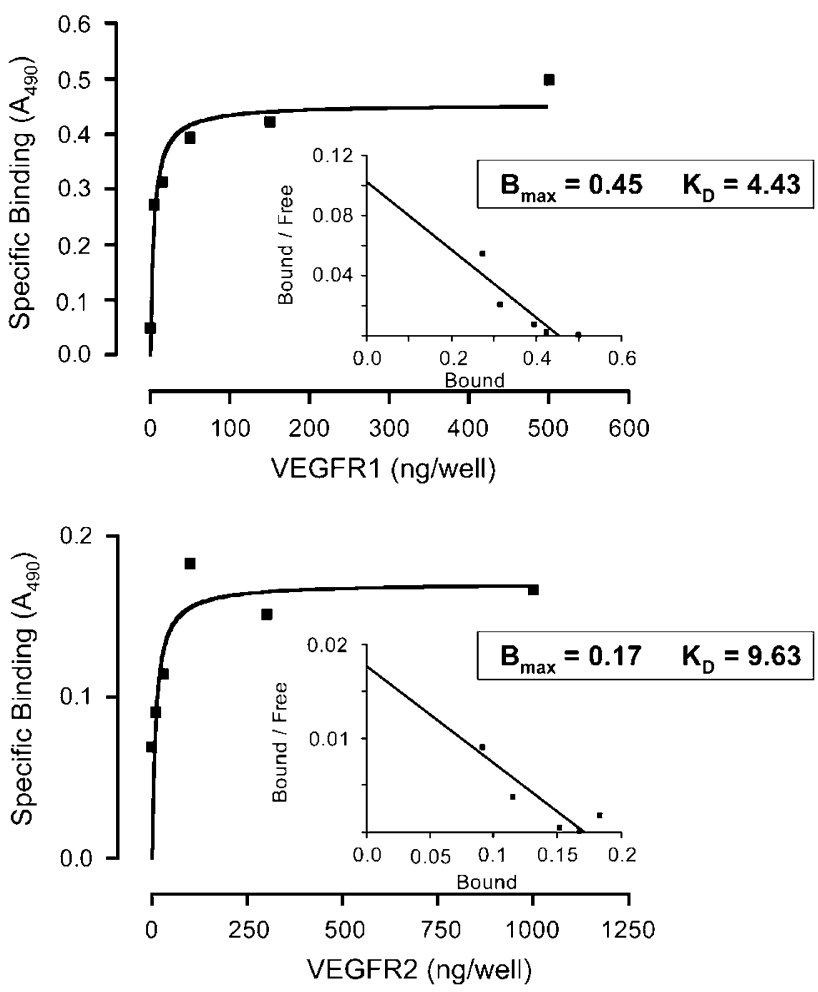

Figure 1 Binding of EMAP II to VEGFR1 and R2 by ELISA. The assay was performed using $800 \mathrm{nM}$ EMAP II per well for precoating, and incubating with increasing concentrations $(1,3,10,30$ and $100 \mathrm{ng} / \mathrm{ml})$ of VEGF R1 or R2. Binding was detected by chromogenic ELISA and absorbance at $490 \mathrm{~nm}$ was plotted as a function of bound VEGFR1 or R2. A dose-dependent increase in binding of EMAP II to VEGFR1 and R2 was observed. Data are expressed as the mean \pm s.d. $(n=6)$.

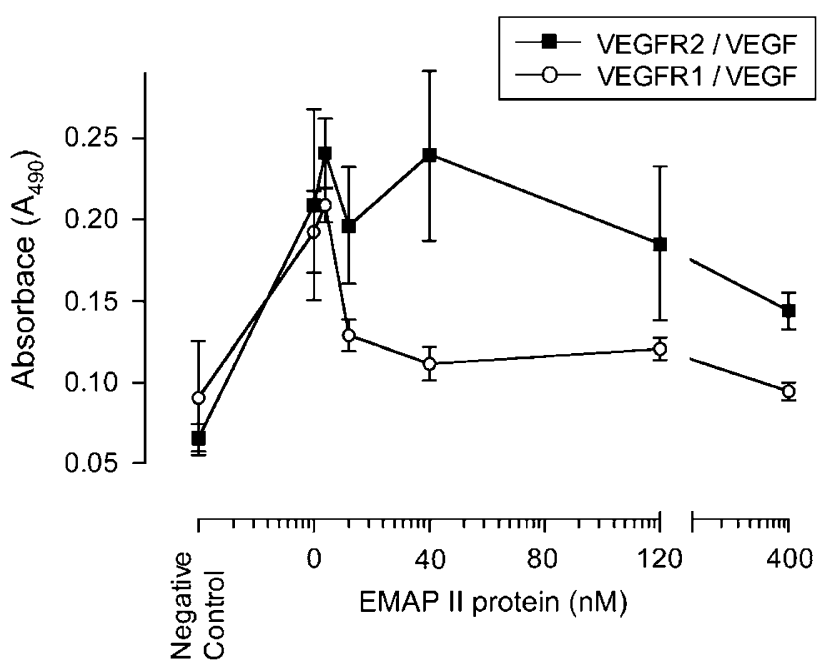

Figure 2 Effect of EMAP II on binding of VEGF to VEGFR1 or R2 was analyzed by ELISA. The assay was performed by preincubating $50 \mathrm{ng} / \mathrm{ml}$ of VEGFR1 or R2 with increasing concentrations $(4,40,120$ and $400 \mathrm{nM})$ of EMAP II for $30 \mathrm{~min}$ followed by addition onto 96-well plate precoated with $250 \mathrm{ng} / \mathrm{ml}$ of VEGF. The binding was detected by chromogenic ELISA, and absorbance at $490 \mathrm{~nm}$ was plotted as a function of bound VEGFR1 or R2. Data are expressed as the mean \pm s.d. $(n=6)$. NC $=$ negative control (no VEGFR added).
For these experiments, a 10-min incubation with VEGF was selected, as we had observed downstream VEGF signaling protein activation peaks at $10-20 \mathrm{~min}$ and decreases at 30 min (data not shown). In HFLECs, we first verified the expression levels of VEGFR1 and VEGFR2 by western blot analysis (Figure 3a). VEGF treatment $(20 \mathrm{ng} / \mathrm{ml})$ for $10 \mathrm{~min}$ increased the phosphorylation of VEGFR1 more than ninefold. EMAP II pretreatment for $30 \mathrm{~min}$ decreased the VEGF-induced phosphorylation of VEGFR1 by $>50 \%$ (Figure 3a). No band for phospho-VEGFR2 was detected in the HFLEC lysate with the antibody used (data not shown).

In HUVECs, EMAP II pretreatment significantly decreased the VEGF-induced phosphorylation of both receptors. As shown in Figure 3b, when the expression of phosphoVEGFR1 and phospho-VEGFR2 was normalized against total VEGFR1 and VEGFR2 expression levels, densitometric analysis showed that VEGF increased phospho-VEGFR1 expression by more than twofold, but EMAP II pretreatment reduced this to levels below the control. Phospho-VEGFR2 was below the detection limit in control cells, but noticeable expression was induced by VEGF treatment; this induction was inhibited by approximately $67 \%$ when cells were pretreated with EMAP II (Figure 3b). The expression of the reference gene $\alpha$-tubulin was not affected by VEGF or EMAP II treatment.

\section{EMAP II Blocks Phosphorylation of VEGF-Induced Signaling Proteins}

To determine the functional aspects of binding between EMAP II and VEGFR1 or VEGFR2, and the observed attenuation of VEGF receptor phosphorylation by EMAP II, we examined the effect of EMAP II on VEGF-mediated phosphorylation of its known downstream signaling proteins Akt, Erk1/2, p38 and Raf. For this experiment, cells were incubated with basal EC medium for $5 \mathrm{~h}$ before treatment with EMAP II and VEGF. As shown in Figure 4a, VEGF (20 ng/ml) treatment for 10 and $20 \mathrm{~min}$ significantly increased the phosphorylation of Akt, Erk1/2, p38 and Raf 2.8-, 1.5-, 2. 2- and 3.6-fold, respectively, as determined by densitometric quantitation. EMAP II treatment alone caused no significant change in expression of these proteins compared with control. However, EMAP II pretreatment blocked the VEGFmediated increase in phosphorylation of Akt, Erk1/2, p38 and Raf, with levels of these phosphorylated proteins either equal to or below the control levels (Figure $4 \mathrm{a}$ and $\mathrm{b}$ ). For the quantitation of protein levels of phosphorylated Akt, Erk1/2, p38 and Raf, all blot signals had been normalized with their corresponding total protein signals that had shown no changes with VEGF and/or EMAP II treatment.

\section{EMAP II Inhibits VEGF-Induced Cell Proliferation}

Next, we tested the effect of EMAP II on VEGF-induced proliferation of ECs in vitro. In HUVECs, FBS (10\%) and reconstituted E-Stim medium was used as positive control for the induction of cellular proliferation. We observed a 
a
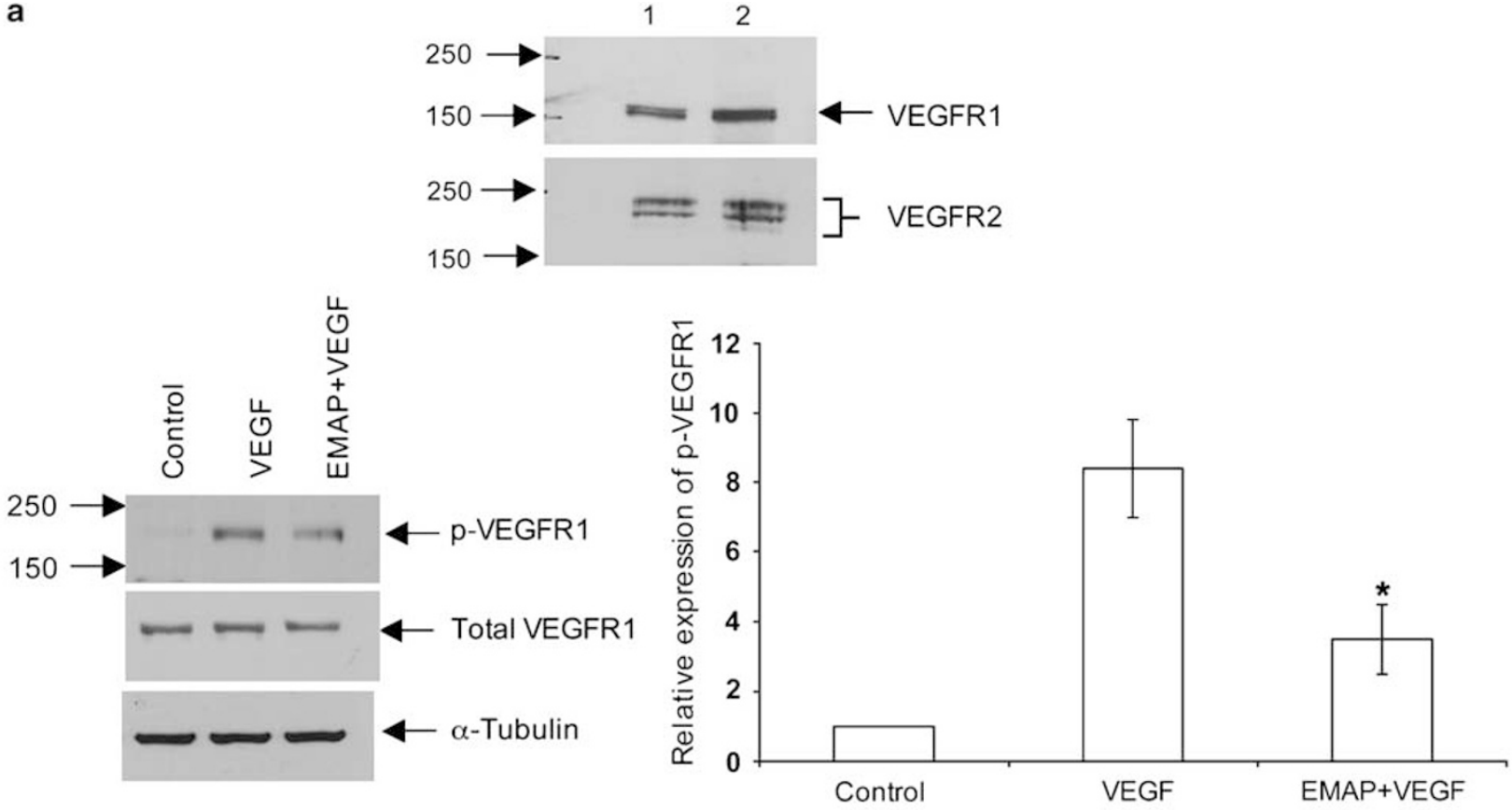

b
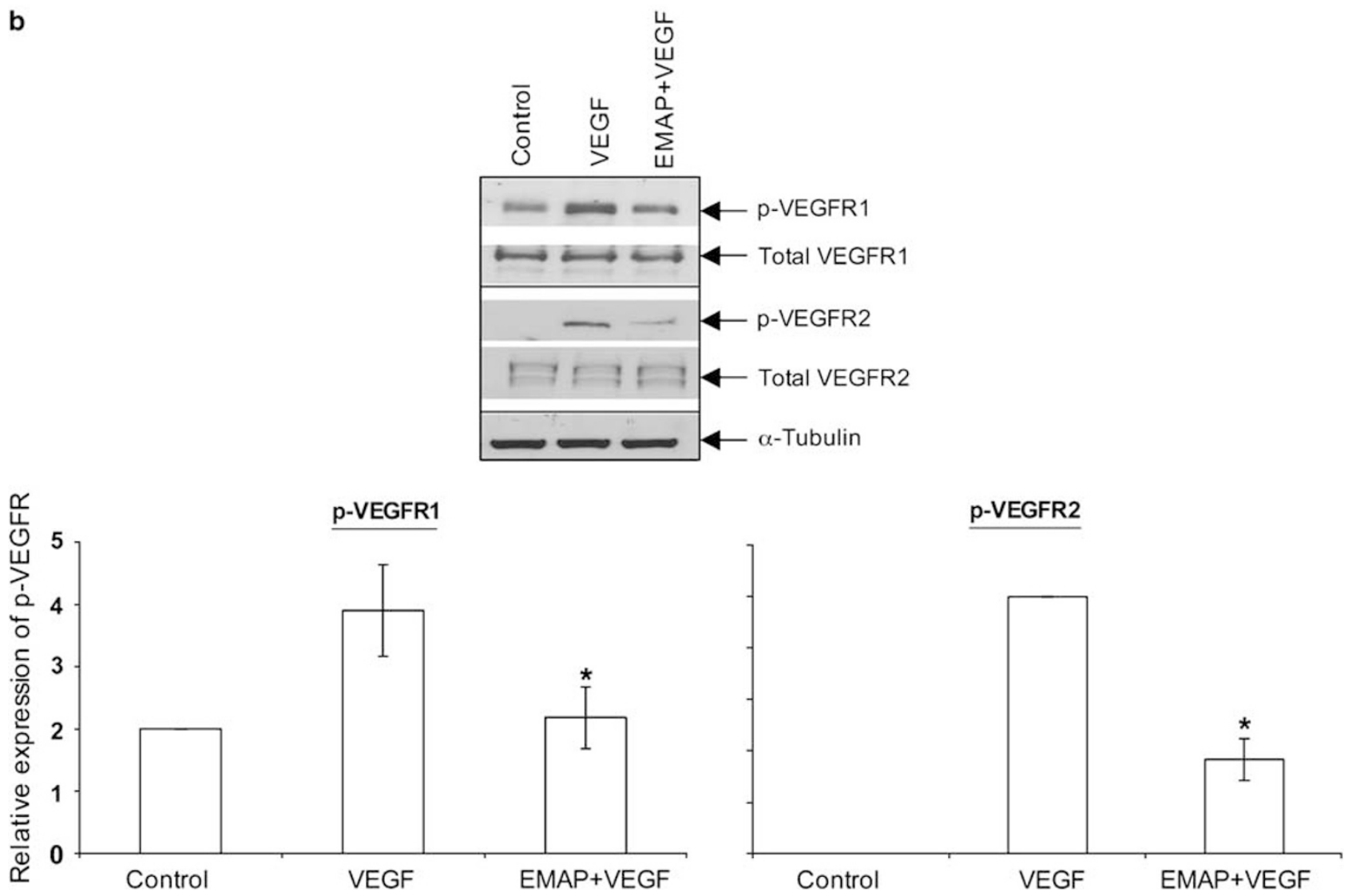

Figure 3 (a) Upper panel shows the western blot analysis for expression of VEGFR1 and VEGFR2 protein in HFLEC. The total protein amount loaded onto the gel was $40 \mu \mathrm{g}$ (lane 1) and $80 \mu \mathrm{g}$ (lane 2). The lower panel shows a western blot analysis for activation of VEGFR1 in HFLEC incubated with $20 \mathrm{ng} / \mathrm{ml}$ VEGF $(10 \mathrm{~min})$, or pretreated with $20 \mu \mathrm{M}$ EMAP II and then treated with $20 \mathrm{ng} / \mathrm{ml}$ VEGF. Immunoblotting was performed using total cell lysate and phosphoVEGFR1 antibody. The intensity of bands was quantitated by densitometry and represented as bar graph. Data are the mean \pm s.d. of three independent experiments. ${ }^{*}$ represents a significant difference from VEGF treatment alone. (b) The upper panel shows the western blot analysis for activation of VEGFR1 and R2 in HUVEC incubated with $20 \mathrm{ng} / \mathrm{ml} \mathrm{VEGF}(10 \mathrm{~min})$, or pretreated with $20 \mu \mathrm{M}$ EMAP II followed by treatment with $20 \mathrm{ng} / \mathrm{ml}$ VEGF. Immunoblotting was performed using total cell lysate and phospho-VEGFR1 and phospho-VEGFR2 antibodies. The lower panel shows the graphical representation of bands quantitated by densitometry. Data are the mean \pm s.d. of three independent experiments. ${ }^{\star}$ represents a significant difference from VEGF treatment alone. 
a

$\begin{array}{llllllll}\text { VEGF }(20 \mathrm{ng} / \mathrm{ml}) & - & 10^{\prime} & 20^{\prime} & - & - & 10^{\prime} & 20^{\prime} \\ \text { EMAPII }(20 \mu \mathrm{M}) & - & - & - & 30^{\prime} & 12 \mathrm{~h} & 30^{\prime} & 30^{\prime}\end{array}$

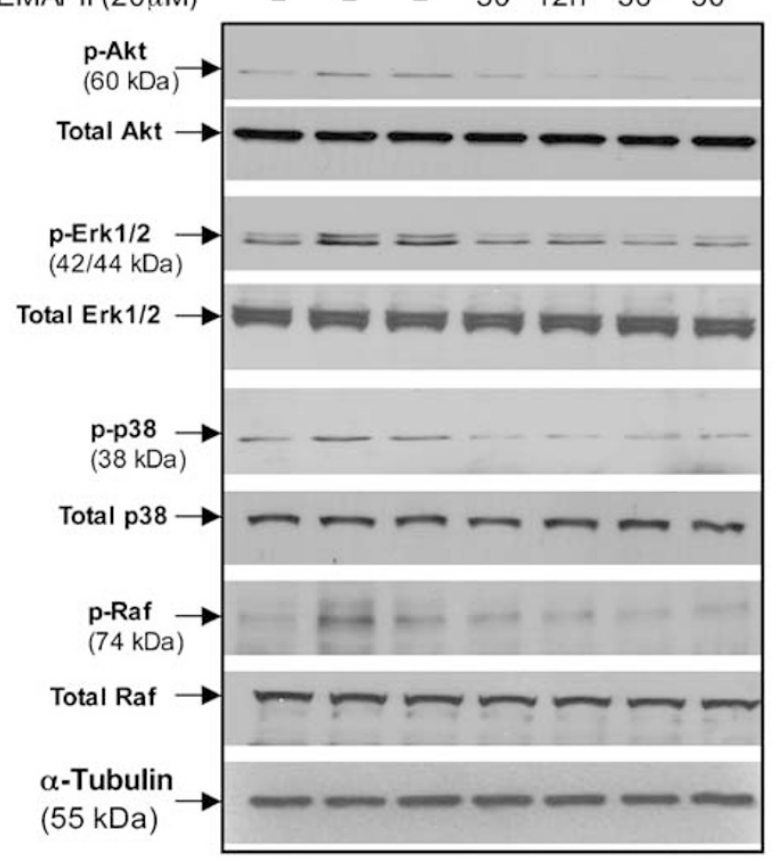

b

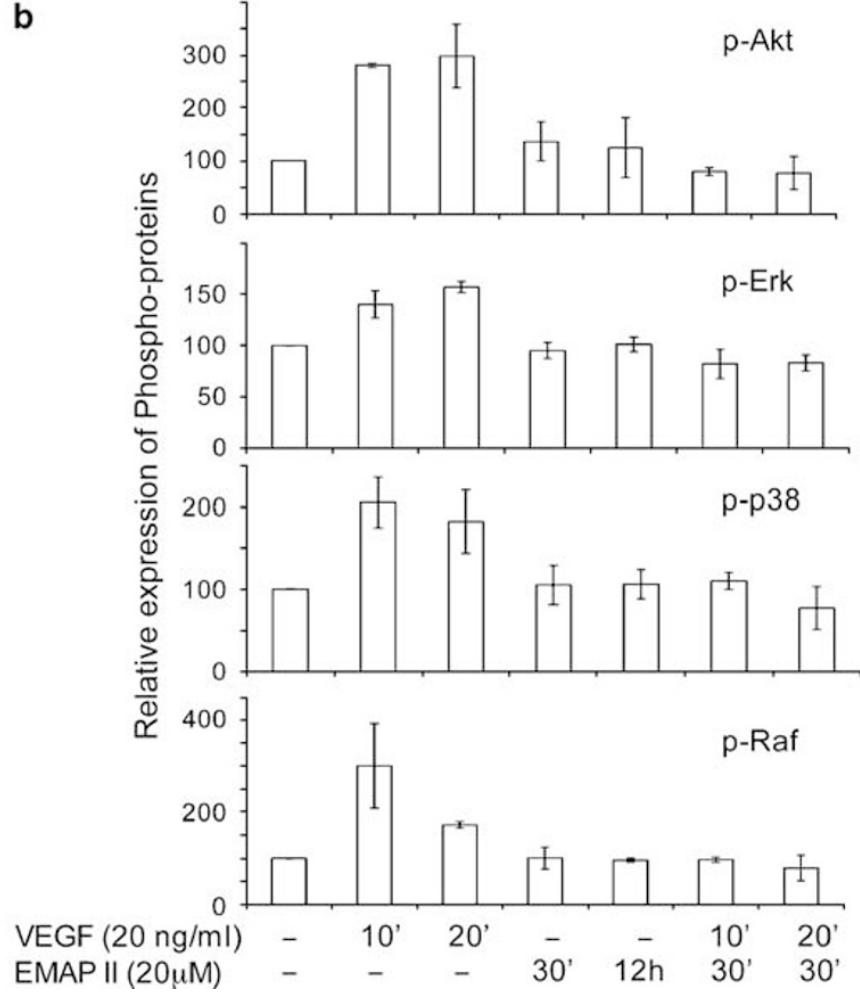

Figure 4 Western blot analysis for activation of Akt, Erk, p38 and Raf protein in HFLEC incubated with $20 \mathrm{ng} / \mathrm{ml} \mathrm{VEGF} \mathrm{(10} \mathrm{or} 20 \mathrm{~min}$ ), $20 \mu \mathrm{M} \mathrm{EMAP} \mathrm{II} \mathrm{(30} \mathrm{min}$ or $12 \mathrm{~h}$ ), EMAP II (30 min) + VEGF (10 min) or EMAP II (30 min) + VEGF (20 min). (a) Total cell lysate was prepared, proteins were separated by SDS-PAGE and immunoblotting was performed using corresponding phospho-specific antibodies. Data are representative of two independent experiments with identical conditions. (b) The intensity of bands was quantitated by densitometry and is represented in the bar graph. Data are the mean \pm s.d. of two independent experiments with identical conditions.

dose-dependent increase in viable cell numbers over time through VEGF treatment. At a $20 \mathrm{ng} / \mathrm{ml}$ concentration, VEGF induced the cell growth by a factor of 2.5 compared to controls after $72 \mathrm{~h}$ incubation. EMAP II treatment alone did not have a significant effect on EC proliferation, but it significantly blocked the VEGF-induced proliferation, as the resulting numbers of viable cells in the EMAP II + VEGF group was very comparable to controls (Figure 5). Similar results were observed in HFLECs where $20 \mathrm{ng} / \mathrm{ml}$ VEGF increased the cell proliferation 1.7-fold, and EMAP II pretreatment abrogated this effect (data not shown).

\section{EMAP II Reduces VEGF-Induced Cell Migration}

The effect of EMAP II on VEGF-induced migration of HUVECs was examined in a transwell chamber assay. As shown in Figure 6, when VEGF alone was used as chemoattractant, migration of HUVECs was increased by $>2.5$-fold, as compared with negative controls. EMAP II alone caused no significant effect on cell migration, but when cells were pretreated with EMAP II, VEGF-induced migration was significantly inhibited and the resulting numbers of migratory cells were very comparable to negative controls.

\section{DISCUSSION}

Three factors were derived from supernatants of cultured murine tumor cells that have capability to induce tissue factor on ECs. These were termed EMAP I-III and among these, EMAP I and II were novel cytokines, ${ }^{1}$ whereas EMAP III turned out to be identical to the previously described VEGF. $^{29}$ EMAP II is a now well-characterized proinflammatory cytokine that inhibits tumor growth and metastasis by inhibition of angiogenesis. However, the precise signaling pathways for antiangiogenic activity of EMAP II have not yet been fully elucidated. In a previous study in our laboratory, we observed that EMAP II caused a significant reduction of VEGF expression in tumor tissues. ${ }^{13}$ In addition to tumor cell-derived VEGF driving tumor progression through proangiogenic mechanisms, VEGF signaling has been reported to be regulated by autocrine or paracrine regulatory loops. ${ }^{30,31}$ In this study, we now demonstrate that EMAP II interacts directly with the VEGF receptors VEGFR1 and VEGFR2, which suggests a possible mechanism for the observed VEGF depletion and reduction in tumor growth. The binding affinity of EMAP II was higher for VEGFR1 compared to VEGFR2. EMAP II competitively inhibited the binding of VEGF to VEGFR1 and VEGFR2, and this inhibition again was greater for VEGFR1 compared to 


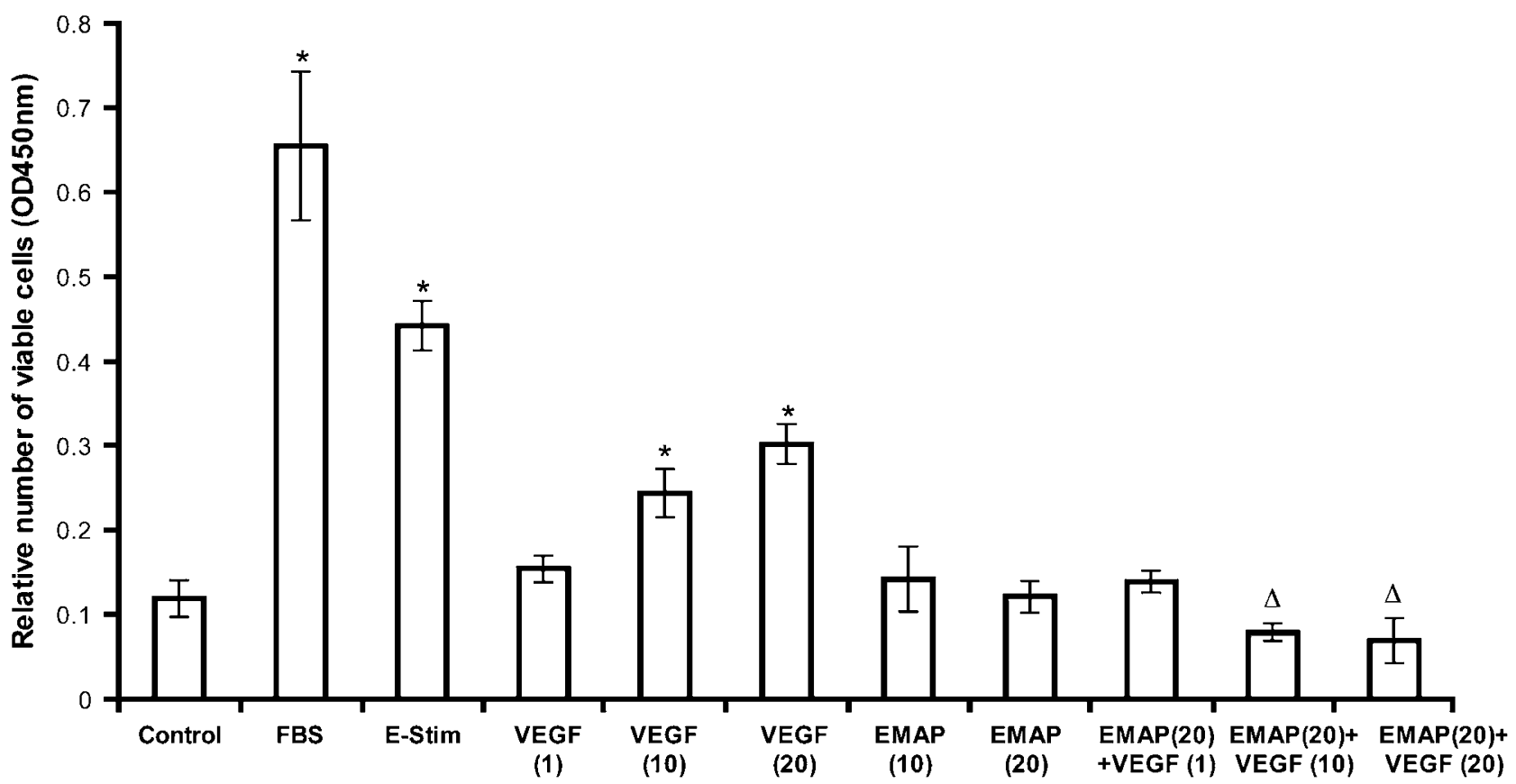

Figure 5 EMAP II inhibits the VEGF-induced proliferation of HUVEC. In a 96-well microplate, cells in basal endothelial cell medium (EBM-2) were treated with VEGF $(1,10$ or $20 \mathrm{ng} / \mathrm{ml})$ and EMAP II $(10$ or $20 \mu \mathrm{M})$. In cotreated samples, cells were pretreated with $20 \mu \mathrm{M}$ EMAP II for 30 min and then treated with VEGF. FBS (10\%) containing EBM-2 medium and E-Stim medium were used as positive control for HUVEC cell proliferation. After $72 \mathrm{~h}$ incubation, cell proliferation was evaluated using the colorimetric WST-1 assay. Data are the mean \pm s.d. of triplicate determinations. The markers indicate a significant difference in the number of viable cells compared with controls $(*)$ and VEGF alone $(\Delta)$, respectively.

VEGFR2. Interestingly, binding affinity of VEGF with VEGFR1 has been reported to be higher than compared to that with VEGFR2. ${ }^{32}$ VEGFR1 and VEGFR2 share structural similarities, and there is a $43.2 \%$ overall sequence homology. Between VEGFR1 and VEGFR2, the extracellular domain homology is $33.3 \%$ and cytoplasmic region homology is $54.6 \%$, whereas kinase domain is the most conserved region with $70.1 \%$ homology. ${ }^{33}$ We do not know where exactly EMAP II binds to the VEGFRs, but suspect that this binding is the cardinal event in the mediated inhibition of VEGFrelated signaling events. In addition, whereas our ELISA data support specific binding between EMAP II and VEGFR, steric hindrance cannot be entirely excluded to partake in this inhibitory effect.

Recent studies to evaluate the antiangiogenic mechanism of EMAP II on ECs revealed that EMAP II also binds with $\alpha$-ATP synthase ${ }^{34}$ and $\alpha 5 \beta 1$ integrin. $^{7}$ However, this does not easily explain any reduction in intratumoral VEGF expression as observed under EMAP II treatment. ${ }^{13}$ The role of paracrine VEGF signaling is well established in the process of angiogenesis, and recently an autocrine VEGF signaling regulatory loop has been proposed to be important for vascular homeostasis. $^{30,31}$ The binding of EMAP II and VEGF receptors may therefore cause interference in either autocrine and/or paracrine VEGF regulatory loops and thus may lead to downregulation of VEGF expression.

VEGF be involved in angiogenesis-induced tumor growth. VEGF exerts most of its biological effects by binding with
VEGFR1 and VEGFR2, which drives receptor dimerization and transphosphorylation on key tyrosine residues, initiating downstream cytoplasmic signaling. VEGFR2 is implicated in most of the VEGF-induced angiogenic processes, whereas the role of VEGFR1 signaling in this process is less well defined. VEGFR1 has been considered as a decoy receptor that can modulate VEGFR2 signaling. ${ }^{35}$ Recent studies have shown that small molecules inhibiting VEGF receptor activation result in inhibition of tumor growth. ${ }^{36,37}$ In our studies, we also observed that EMAP II pretreatment resulted in a significant decrease in VEGF-induced phosphorylation of VEGFR1 and VEGFR2 in HUVECs. In HFLECs, EMAP II caused a similar effect on VEGFR1 activation but we could not observe any signal for phospho-VEGFR2. The phosphoVEGFR2 antibody used in these experiments was specific for detecting phosphorylation at Tyr1054, therefore either there is no phosphorylation of VEGFR2 at Tyr1054 in HFLECs, or the expression levels are too low to detect. In HFLECs, we also used phospho-VEGFR2 antibody specific for detecting phosphorylation at Try1175 and Tyr951 but we could not detect any signal on western blot.

Previous reports have shown that activation of VEGF receptors leads to the activation of Raf and the downstream Erk1/2 (p42/44) mitogen-activated kinases, which is involved in cell proliferation. ${ }^{38-40}$ VEGF also activates PI3-kinase/Akt signal transduction pathway, which has been implicated in EC survival. ${ }^{41}$ Also, VEGF activates the p38 MAPK pathway that is required for mitogenic activity in ECs and has been 


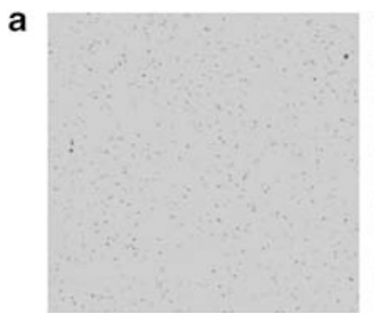

Control

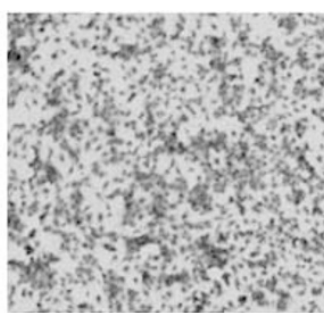

FBS

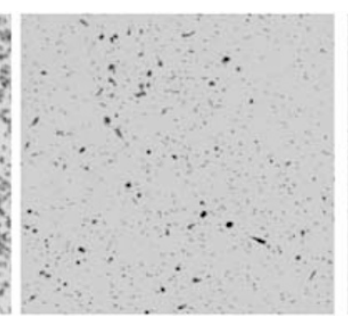

VEGF

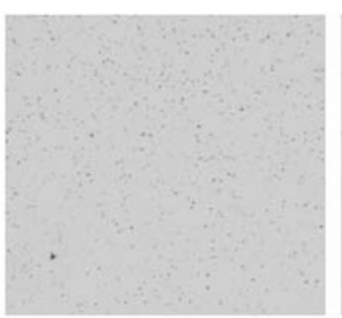

EMAP

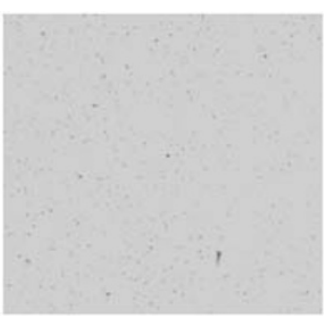

EMAP+VEGF

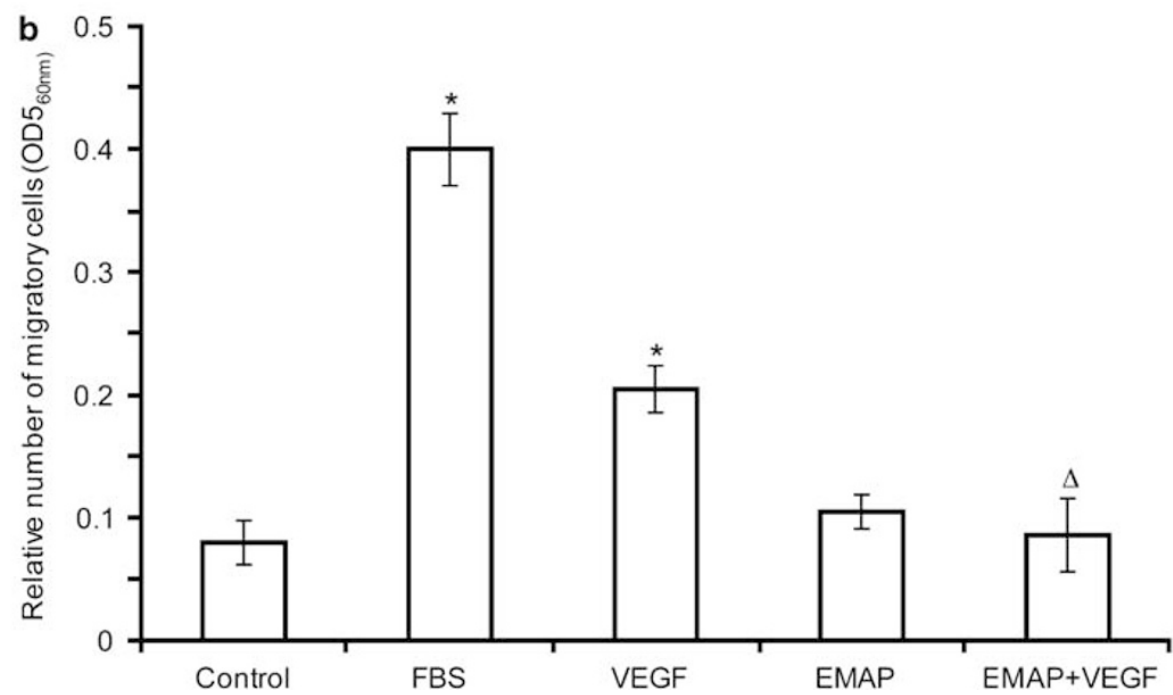

Figure 6 EMAP II inhibits VEGF-induced migration of endothelial cells. In 24-well plates, the upper polycarbonate membrane insert contained HUVECs suspended in EBM-2 medium with or without EMAP II pretreatment. In the lower chamber, $10 \%$ FBS, $20 \mathrm{ng} / \mathrm{ml}$ VEGF or $20 \mu \mathrm{M}$ EMAP II were added in $500 \mu \mathrm{l}$ serum-free endothelial cell medium, and the plate was incubated for $24 \mathrm{~h}$. (a) Migratory cells on the bottom of the polycarbonate membrane were stained and photographed using inverted light microscope. Photographs shown are representative from one of two independent experiments with each sample in triplicate. (b) Stained migratory cells were extracted and quantitated by absorption at $560 \mathrm{~nm}$. Data are the mean \pm s.d. of triplicate determination. The markers indicate a significant difference in the number of migratory cells compared with controls $(*)$ and VEGF alone $(\Delta)$, respectively.

implicated in cell migration. ${ }^{42,43}$ In our study, we observed that EMAP II preincubation inhibited VEGF-induced activation of elements of all these downstream signaling pathways, namely Raf, Erk1/2, Akt and p38. This inhibition of phosphorylation of VEGF-induced cytoplasmic signaling proteins likely reflects a functional interference with the VEGF to VEGFR interaction, and represents a mechanism for the known effects of EMAP II on survival, proliferation, differentiation and migration of ECs.

VEGF-induced proliferation of ECs be involved in angiogenesis-induced tumor growth. ${ }^{17}$ Several studies have shown that VEGF induces proliferation of ECs. ${ }^{44}$ Also, inhibition of ECs proliferation has been suggested for antiangiogenic activity of EMAP II. ${ }^{6,8,11}$ In our study, we observed that EMAP II preincubation significantly inhibited the VEGF-induced ECs proliferation. This reduction in VEGF-induced cell proliferation is in good agreement with our finding that EMAP II inhibits VEGF-induced activation of Erk1/2, which is a major downstream target of VEGFR2, known to be involved in cell proliferation. In addition, VEGF has been shown to be a potent stimulator of EC migration, ${ }^{45}$ a major contributor to angiogenesis-induced tumor growth. Studies have shown that EMAP II acts as chemoattractant for neutrophils and monocytes. ${ }^{2}$ In our studies no significant change in HUVEC migration was observed when EMAP II alone was used as chemoattractant, but when HUVECs were pretreated with EMAP II, the VEGF-induced migration of cells was inhibited. This finding correlates with our observation that EMAP II blocks VEGF-induced activation of p38 MAPK, which has been implicated in EC migration. ${ }^{42,43}$

In summary, our study demonstrated that EMAP II interacts with VEGF receptors, which inhibits VEGF receptor activation and causes inhibition of VEGF-induced activation of downstream signaling proteins. We also showed that EMAP II inhibits VEGF-induced ECs proliferation and migration. This heretofore unknown mechanism of EMAP II may provide an explanation for its in vivo antitumor activity, and may also suggest therapeutic strategies for the enhancement of anti-VEGF therapy to inhibit angiogenesisdriven tumor growth.

1. Kao J, Ryan J, Brett G, et al. Endothelial monocyte-activating polypeptide II. A novel tumor-derived polypeptide that activates host-response mechanisms. J Biol Chem 1992;267:20239-20247. 
2. Kao J, Houck K, Fan Y, et al. Characterization of a novel tumor-derived cytokine. Endothelial-monocyte activating polypeptide II. J Biol Chem 1994;269:25106-25119.

3. van Horssen R, Eggermont AM, ten Hagen TL. Endothelial monocyteactivating polypeptide-II and its functions in (patho)physiological processes. Cytokine Growth Factor Rev 2006;17:339-348.

4. Barnett $G$, Jakobsen $A M$, Tas $M$, et al. Prostate adenocarcinoma cells release the novel proinflammatory polypeptide EMAP-II in response to stress. Cancer Res 2000;60:2850-2857.

5. Liu J, Schwarz MA. Identification of protease-sensitive sites in human endothelial-monocyte activating polypeptide II protein. Exp Cell Res 2006;312:2231-2237.

6. Tandle AT, Mazzanti C, Alexander HR, et al. Endothelial monocyte activating polypeptide-II induced gene expression changes in endothelial cells. Cytokine 2005;30:347-358.

7. Schwarz MA, Zheng $\mathrm{H}$, Liu J, et al. Endothelial-monocyte activating polypeptide II alters fibronectin based endothelial cell adhesion and matrix assembly via alpha5 beta1 integrin. Exp Cell Res 2005;311:229-239.

8. Wu PC, Alexander HR, Huang J, et al. In vivo sensitivity of human melanoma to tumor necrosis factor (TNF)-alpha is determined by tumor production of the novel cytokine endothelial-monocyte activating polypeptide II (EMAP II). Cancer Res 1999;59:205-212.

9. Gnant MF, Berger $A C$, Huang J, et al. Sensitization of tumor necrosis factor alpha-resistant human melanoma by tumor-specific in vivo transfer of the gene encoding endothelial monocyte-activating polypeptide II using recombinant vaccinia virus. Cancer Res 1999;59:4668-4674.

10. Berger AC, Alexander HR, Wu PC, et al. Tumour necrosis factor receptor I (p55) is upregulated on endothelial cells by exposure to the tumourderived cytokine endothelial monocyte-activating polypeptide II (EMAP-II). Cytokine 2000;12:992-1000.

11. Schwarz MA, Kandel J, Brett J, et al. Endothelial-monocyte activating polypeptide II, a novel antitumor cytokine that suppresses primary and metastatic tumor growth and induces apoptosis in growing endothelial cells. J Exp Med 1999;190:341-354.

12. Berger AC, Alexander HR, Tang G, et al. Endothelial monocyte activating polypeptide II induces endothelial cell apoptosis and may inhibit tumor angiogenesis. Microvasc Res 2000;60:70-80.

13. Schwarz RE, Schwarz MA. In vivo therapy of local tumor progression by targeting vascular endothelium with EMAP-II. J Surg Res 2004;120:64-72.

14. Hanahan D, Folkman J. Patterns and emerging mechanisms of the angiogenic switch during tumorigenesis. Cell 1996;86:353-364.

15. Folkman J. Angiogenesis in cancer, vascular, rheumatoid and other disease. Nat Med 1995;1:27-31.

16. Folkman J, Shing Y. Angiogenesis. J Biol Chem 1992;267: 10931-10934.

17. Jakeman LB, Winer J, Bennett GL, et al. Binding sites for vascular endothelial growth factor are localized on endothelial cells in adult rat tissues. J Clin Invest 1992;89:244-253.

18. Senger DR, Perruzzi CA, Feder J, et al. A highly conserved vascular permeability factor secreted by a variety of human and rodent tumor cell lines. Cancer Res 1986;46:5629-5632.

19. Risau W. Mechanisms of angiogenesis. Nature 1997;386:671-674.

20. Kim KJ, Li B, Winer J, et al. Inhibition of vascular endothelial growth factor-induced angiogenesis suppresses tumour growth in vivo. Nature 1993;362:841-844.

21. Ferrara N. Vascular endothelial growth factor: molecular and biological aspects. Curr Top Microbiol Immunol 1999;237:1-30.

22. Alon T, Hemo I, Itin A, et al. Vascular endothelial growth factor acts as a survival factor for newly formed retinal vessels and has implications for retinopathy of prematurity. Nat Med 1995;1:1024-1028.

23. Millauer $\mathrm{B}$, Longhi MP, Plate $\mathrm{KH}$, et al. Dominant-negative inhibition of Flk-1 suppresses the growth of many tumor types in vivo. Cancer Res 1996;56:1615-1620.

24. Goldman CK, Kendall RL, Cabrera G, et al. Paracrine expression of a native soluble vascular endothelial growth factor receptor inhibits tumor growth, metastasis, and mortality rate. Proc Natl Acad Sci USA 1998;95:8795-8800.

25. Prewett $\mathrm{M}$, Huber J, Li Y, et al. Antivascular endothelial growth factor receptor (fetal liver kinase 1) monoclonal antibody inhibits tumor angiogenesis and growth of several mouse and human tumors. Cancer Res 1999;59:5209-5218.

26. Shibuya M, Ito N, Claesson-Welsh L. Structure and function of vascular endothelial growth factor receptor-1 and -2. Curr Top Microbiol Immunol 1999;237:59-83.

27. Zachary I. VEGF signalling: integration and multi-tasking in endothelial cell biology. Biochem Soc Trans 2003;31:1171-1177.

28. Schwarz MA, Zhang F, Gebb S, et al. Endothelial monocyte activating polypeptide II inhibits lung neovascularization and airway epithelial morphogenesis. Mech Dev 2000;95:123-132.

29. Clauss $M$, Gerlach $M$, Gerlach $H$, et al. Vascular permeability factor: a tumor-derived polypeptide that induces endothelial cell and monocyte procoagulant activity, and promotes monocyte migration. J Exp Med 1990;172:1535-1545.

30. Augustin HG. Antiangiogenic tumour therapy: will it work? Trends Pharmacol Sci 1998;19:216-222.

31. Lee $S$, Chen $T$, Barber $C L$, et al. Autocrine VEGF signaling is required for vascular homeostasis. Cell 2007;130:691-703.

32. Terman BI, Dougher-Vermazen M, Carrion ME, et al. Identification of the KDR tyrosine kinase as a receptor for vascular endothelial cell growth factor. Biochem Biophys Res Commun 1992;187:1579-1586.

33. Rahimi N. Vascular endothelial growth factor receptors: molecular mechanisms of activation and therapeutic potentials. Exp Eye Res 2006;83:1005-1016.

34. Chang SY, Park SG, Kim S, et al. Interaction of the C-terminal domain of p43 and the alpha subunit of ATP synthase. Its functional implication in endothelial cell proliferation. J Biol Chem 2002;277:8388-8394.

35. Ferrara N, Gerber HP, LeCouter J. The biology of VEGF and its receptors. Nat Med 2003;9:669-676.

36. Lu N, Yang Y, You QD, et al. Gambogic acid inhibits angiogenesis through suppressing vascular endothelial growth factor-induced tyrosine phosphorylation of KDR/Flk-1. Cancer Lett 2007;258:80-89.

37. Smith NR, James NH, Oakley l, et al. Acute pharmacodynamic and antivascular effects of the vascular endothelial growth factor signaling inhibitor AZD2171 in Calu-6 human lung tumor xenografts. Mol Cancer Ther 2007;6:2198-2208.

38. Kroll J, Waltenberger J. The vascular endothelial growth factor receptor KDR activates multiple signal transduction pathways in porcine aortic endothelial cells. J Biol Chem 1997;272:32521-32527.

39. Sa G, Fox PL. Basic fibroblast growth factor-stimulated endothelial cell movement is mediated by a pertussis toxin-sensitive pathway regulating phospholipase A2 activity. J Biol Chem 1994;269:32193225 .

40. Cross MJ, Dixelius J, Matsumoto T, et al. VEGF-receptor signal transduction. Trends Biochem Sci 2003;28:488-494.

41. Gerber HP, McMurtrey A, Kowalski J, et al. Vascular endothelial growth factor regulates endothelial cell survival through the phosphatidylinositol 3'-kinase/Akt signal transduction pathway. Requirement for Flk-1/KDR activation. J Biol Chem 1998;273:3033630343.

42. Cezar-de-Mello PF, Nascimento-Silva V, Villela CG, et al. Aspirintriggered Lipoxin A4 inhibition of VEGF-induced endothelial cell migration involves actin polymerization and focal adhesion assembly. Oncogene 2006;25:122-129.

43. Rousseau S, Houle $\mathrm{F}$, Kotanides $\mathrm{H}$, et al. Vascular endothelial growth factor (VEGF)-driven actin-based motility is mediated by VEGFR2 and requires concerted activation of stress-activated protein kinase 2 (SAPK2/p38) and geldanamycin-sensitive phosphorylation of focal adhesion kinase. J Biol Chem 2000;275:10661-10672.

44. Olsson AK, Dimberg A, Kreuger J, et al. VEGF receptor signaling-in control of vascular function. Nat Rev Mol Cell Biol 2006;7:359-371.

45. Carmeliet $P$, Ferreira V, Breier G, et al. Abnormal blood vessel development and lethality in embryos lacking a single VEGF allele. Nature 1996;380:435-439. 